\title{
Intelligent Optimization of Urban real-time traffic route based on Improved non-dominated Sorting Genetic Algorithm
}

\author{
Zhu Min ${ }^{1,2}$,Su Bo ${ }^{3}$, Ning GangMin ${ }^{1}$
}

1 Dept. of Biomedical Engineering of Zhejiang University ,Hangzhou Zhejiang, 310058 , China

2 Guizhou Key Laboratory of Agricultural Bioengineering of Guizhou University ,Guizhou Guiyang, 550025, China

3 Guizhou Institute of Technology, Guizhou Guiyang, 550025, China

Keywords: improved non-dominated sorting, genetic algorithm, traffic, route

\begin{abstract}
Urban traffic is a real-time change, so the traditional serial algorithm cannot satisfy the requirement of traffic scale and condition changes. Therefore, this paper proposes a improved non-dominated sorting genetic algorithm for route optimization problem of multi-objects. Firstly, through the analysis of the optimization of the Pareto front solution set and the complexity of the algorithm, we determine the multi-objects problem to be optimized, and then we convert the problem into optimization problem of single-object fitness function, which is named as elitism mechanism. Through the strategy, we can obtain the optimal solution of real-time traffic condition.
\end{abstract}

\section{Introduction}

At present, the effect of traffic guidance after traffic congestion has been recognized, K.Wundcrlich's research shows that travel time can save 3\%-9\% after traffic guidance when heavy traffic occurred ${ }^{[1]}$. The greater the lever of traffic congestion, the greater the efficiency of the vehicle to be guidance ${ }^{[2]}$.

Genetic algorithm(GA) is a more effective measure to solve the large-scale traffic guidance operation, it by simulating DNA population evolution, elite population selection process, making in a short period of time to choose the optimal route possible ${ }^{[3]}$. Routing optimization of intersection is usually a Multi-Objective Optimization problem(MOO), which need set the weight of each objective, and added. Due to the different assumptions of the multi-objective, the traditional genetic algorithm can not simply used weight-adding method as the goal of traffic optimization for preventing premature. To solve these problems, Fonseca and Fleming proposed Multi-Objective Genetic Algorithm (MOGA), the objectives of route optimization are maximum number of vehicles and Minimum number of mean queue length ${ }^{[4]}$. The population diversity of MOGA was controlled by using shared function and niche technology, but the size of the niche has great influence on MOGA $^{[5-6]}$. Zitzler E proposed Non-dominated Sorting Genetic Algorithms(NSGA) ${ }^{[7]}$, which Allows for the existence of multiple different equivalent solutions. The disadvantage is that NSGA has a high computational complexity, the lack of elitism mechanism, and a large dependence on the shared parameters ${ }^{[8]}$.

This paper Improved NSGA (INSGA) for MOO of traffic guidance. First, through fitness function, Pareto solution set, to analysis the individual density, and then convert the problem into optimization problem of single-object fitness function to reduce the complexity of computation.. Finally, we proposed elitism mechanism and identify the optimal individual to improve search performance of the algorithm. 


\section{Algorithm analysis}

Real-time traffic condition has multi-objects, so there are multiple choices for navigation. The traditional static algorithm cannot meet the real-time changes. Therefore, this paper proposes a non-dominated sorting genetic algorithm for MOO. First, through Pareto solution set optimization and algorithm complexity analysis, we determine the multi-objects problem to be optimized. Then we convert the problem into optimization problem of single-object fitness function, namely elitism mechanism, through which we can obtain the optimal solution of real-time traffic condition.

\subsection{The fitness function of Pareto frontier solution set optimized}

Suppose when the evolution generation of individual i reaches $t$, it at the frontier of pareto with set of $1+1$ and sorting value of 1 .

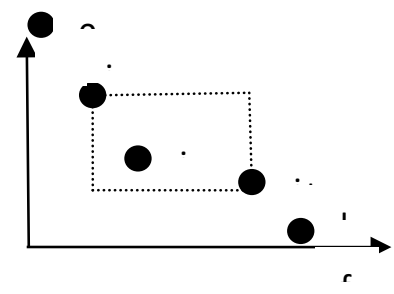

Figure 1 Individual density values calculated figure

The density of individual 0 and 1 at the two ends of the forefront can be described as the following equation

$$
d_{i}^{t}=1 / \sum_{j=1}^{k}\left(f_{j}^{i+1}-f_{j}^{i-1}\right)
$$

It can be inferred from equation 2-1 if the individual distribution is intensive, it has large density value, and if the distribution is spread, the density value is small. The normalized result is $d_{i}=d_{i}^{t} / d_{\max }\left(d_{\max }\right.$ is the maximum value of $\left.d_{i}^{t}\right)$. Taking the density information of $d_{i}$ into Pareto sorting equation, we can obtain the optimized fitness function of individual i:

$$
F_{\text {fit }}(i)=i_{\text {rank }}+\lambda d_{i}
$$

The parameter $\lambda$ in formula 2-2 equals to 0.9 , which ensures the difference of $F_{\text {fit }}(i)$ and $i_{\text {rank }+1}$. The fitness function is obtained by Pareto sorting optimization, so it must be compared with the other individuals according to Pareto sorting principle. The complexity of Pareto is $O\left(N^{2}\right)$, and the density function in this paper is $O(M l \log l)$ wherein $l \leq \mathrm{N}$.

\subsection{Optimization of the elite population}

According to the characters of elite value, it can be reserved keeping $F_{f i t}(i)<2$ in equation $2-2$. In practical applications, one new generation produce a new elite set, if the evolution generation tends to infinity, the size of set approaches infinity. Obviously, it cannot help the decision makers find the most effective elite value. Therefore, this paper proposes an elite optimization tragedy. The 
optimization ideas are as follows:

(1) $F_{f i t}(i)$ can be obtained by formula 2-2, then use bubble sort method to sort.

(2) At the same time, combining with mesh width formulas, we define all individuals in the grid as shown in Figure 2.

$$
g_{w i}=\left[\max f_{i}(x)-\min f_{i}(x)\right] / k_{i}+\zeta, \quad \zeta<10^{-5} \text {, in the formula, } g_{w i} \text { represents the width of }
$$

the i-th dimension of a grid, and $k_{i}$ is the grid number of the i-th dimension.

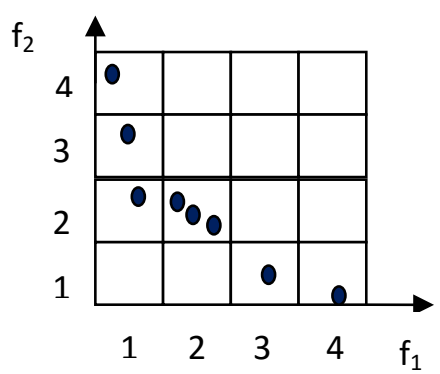

Figure 2 density grid

(3) Scanning points in the grid to determine whether there is a historical optimal point in the current grid. If the current individual is optimal, set as the historical optimal point, and continue the comparison; or else go on the next individual. Through the comparison, we can finally find the only one optimized point.

\section{Simulation}

The paper takes the section from government of Jingyang to Shachong road of Nanming, Guiyang, GuiZhou for example. The length of the section is $16.9 \mathrm{~m}$. Congestion often occurs in the main road, so reasonable diversion and the optimal path can undoubtedly greatly ease the traffic pressure. Take government of Jingyang as Start point (marked with "S"), and Shachong road of Nanming as End point (marked with "E"). The shortest route computed by the traditional static algorithm is marked as the line between $\mathrm{S}$ and $\mathrm{E}$. When there is no real-time traffic information, the simulation result is the same as the one of the static algorithm. When there is real-time traffic information, the optimal route is changing with time. 


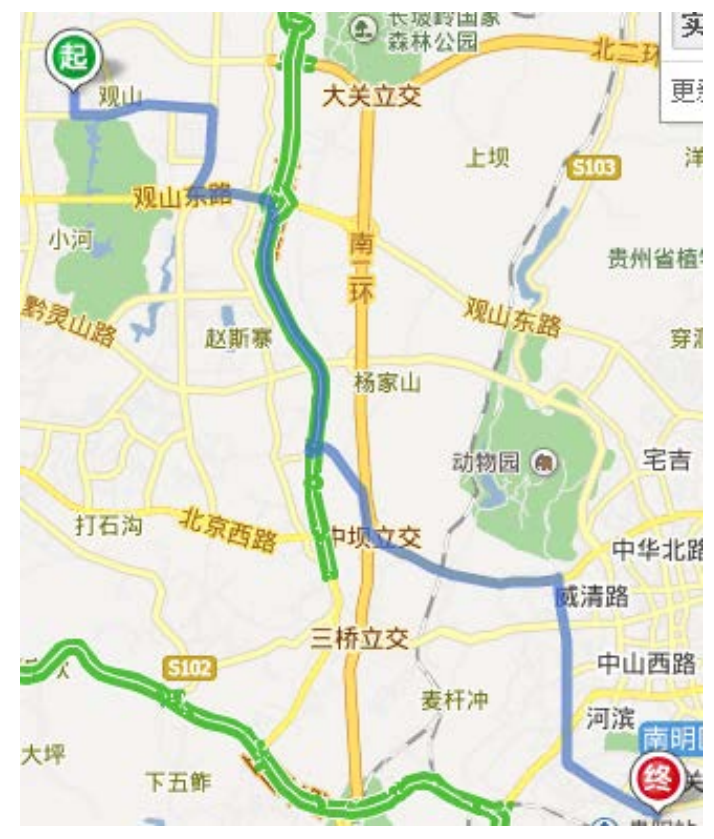

Figure 3 static route

Combining the proposed algorithm with the real-time traffic condition, the optimal route obtained in the simulation is shown in Figure 4.

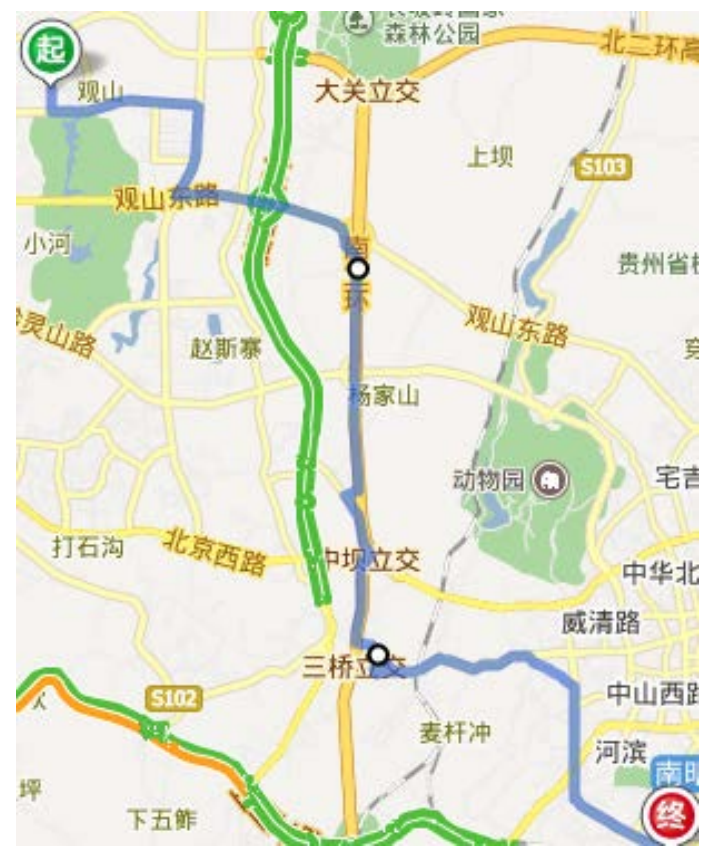

Figure 4 dynamic data

In the current state of the road, according to the static road map, the time-consuming will be 2519 seconds, and by the optimized algorithm, it is only 1857 seconds. In the case of real-time traffic changing, the proposed algorithm will calculate a more optimal path, easing the pressure on the road.

Of course, only one state is not convinced enough, the present study randomly selects other sections, respectively, to simulate the real-time traffic. Finally, experiment results are listed below: 
Table 1 multi-sections route simulation table

\begin{tabular}{|l|l|l|l|l|}
\hline Start and ending section & \multicolumn{4}{|c|}{ Travel time(s) } \\
\hline & $\begin{array}{l}\text { stati } \\
\text { c }\end{array}$ & GA & $\begin{array}{l}\text { NSG } \\
\text { A }\end{array}$ & $\begin{array}{l}\text { INS } \\
\text { GA }\end{array}$ \\
\hline $\begin{array}{l}\text { MiLan Sky- Guizhou Red Cross Women and } \\
\text { Children Hospital }\end{array}$ & 1224 & 1106 & 1098 & 967 \\
\hline Orchard Wetland Park - Huansha Road & 1562 & 1427 & 1204 & 1007 \\
\hline Du Si Lu -Tou Qiao & 1097 & 964 & 932 & 763 \\
\hline Pine Park - Qianling Mansion & 3120 & 2854 & 2617 & 1062 \\
\hline
\end{tabular}

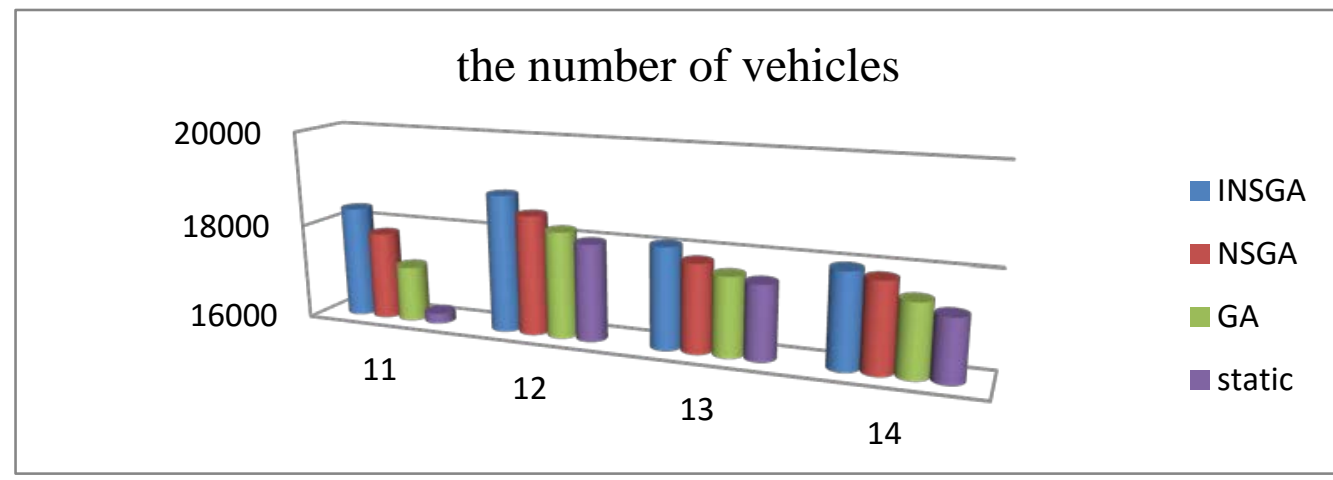

Figure 5 the number of vehicles(11,12,13,14 represent the juctions,11 is between Zhongshan Dong road and Fushui Nan Road,12 is between Zhongshan Dong road and Wenchang Bei Road,13 is between Zhonghua Zhong road and Yanan Road,14 is between Chaoyang Road and Shachong road)

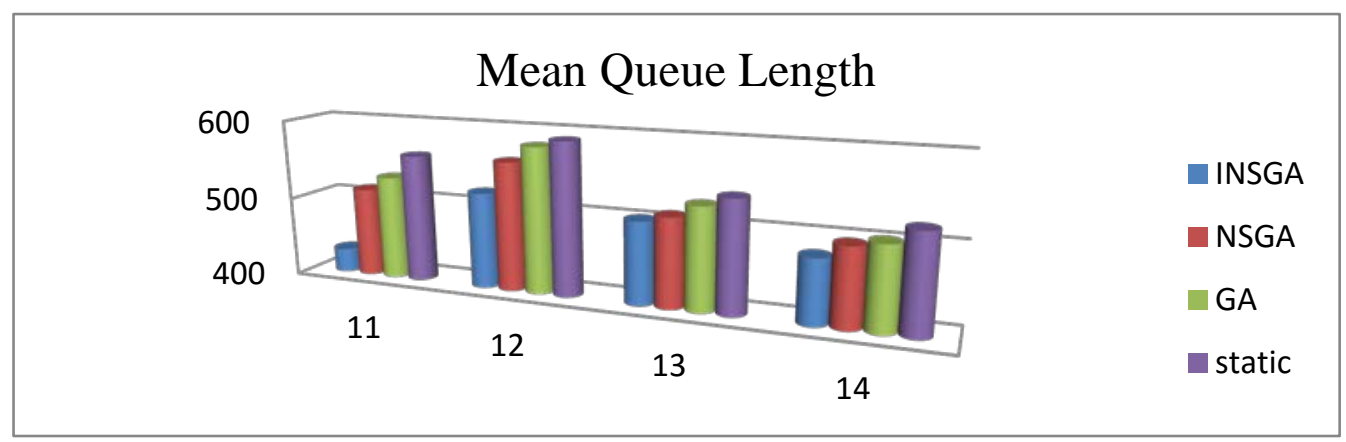

Figure 6 Mean Queue Length

It can be inferred from Table 1 that the algorithm has great effects on the route optimization. As figure5,6 shown, we compared with the two parameters, which are the number and mean queue length of vehicles which passed through the junctions between static, GA, NSGA and INSGA. The first parameter of INSGA is larger than the others algorithms, and the second parameter of INSGA is lower than the others, so we can see the optimization is effective.

\section{Conclusions}

Urban traffic is a real-time change, so the traditional static algorithm cannot satisfy the requirement of intelligent traffic control. Therefore, this paper proposes a non-dominated sorting genetic algorithm for route optimization problem of multi-objects. The simulation verifies its superiority, which may bring some benefit to GuiZhou province.

\section{Acknowledgements}

Science and technology fund projects in guizhou guiyang traffic intelligent control and guidance 
technology research (qian J word [2012] 2012)

\section{References}

[1]James Kennedy, Russell C Eberhart, Swarm Intelligence[M], Posts \& Telecom Press,2012

[2]Eberhart R.C, Computational Intelligence Concepts to Implementations[M], Posts \& Telecom Press,2011

[3]Christian Blum, Swarm Intelligence Introduction and Applications[M], National Defense Industry Press,2012

[4].Fonseca C M.Fleming P J An overview of evolutionary algorithms in multiobjective optimization1995(01)

[5].Chipperfield A.Fleming P Gas Turbine Engine Controller Design using Multiobjective Genetic Algorithms 1995

[6].Todd D S.Sen P A Multiple Criteria Genetic Algorithm for Containership Loading 1997

[7].Zitzler E Evolutionary algorithms for multiobjective optimization:Methods and applications 1999

[8].Zitzler E.Thiele L Multiobjective optimization using evolutionary algorithms-A comparative case study 1998 